\title{
Activation of Cortical and Cerebellar Motor Areas during Executed and Imagined Hand Movements: An fMRI Study
}

\author{
Martin Lotze, Pedro Montoya, Michael Erb, and Ernst Hülsmann \\ University of Tübingen
}

Herta Flor
Humboldt-University Berlin

Uwe Klose, Niels Birbaumer, and Wolfgang Grodd

University of Tübingen

\begin{abstract}
Brain activation during executed (EM) and imagined movements (IM) of the right and left hand was studied in 10 healthy right-handed subjects using functional magnetic resonance imagining (fMRI). Low electromyographic (EMG) activity of the musculi flexor digitorum superficialis and high vividness of the imagined movements were trained prior to image acquisition. Regional cerebral activation was measured by fMRI during EM and IM and compared to resting conditions. Anatomically selected regions of interest (ROIs) were marked interactively over the entire brain. In each ROI activated pixels above a $t$ value of $2.45(p<0.01)$ were counted and analyzed. In all subjects the supplementary motor area (SMA), the premotor cortex (PMC), and the primary motor cortex (M1) showed significant activation during both EM and IM; the somatosen-
\end{abstract}

\section{INTRODUCTION}

The question of what extent imagery and perception share the same neuronal substrates or if they are based on completely different neuronal mechanisms such as abstract, postperceptual representations, has been an ongoing debate in the neurosciences (cf. Farah, 1995; Kosslyn, 1988; Pylyshyn, 1981). This debate has mainly focused on visual imagery, where evidence from neuroimaging and from neuropsychological testing has suggested that imagery and perception utilize the same brain areas. In addition, visual image generation seems to involve areas in the frontal and/or parietal cortex (Farah, 1995; Schupp, Lutzenberger, Birbaumer, Miltner, \& Braun, 1994). Motor imagery has different characteristics than visual imagery: It is not the virtual environment that is imagined but introspective kinesthetic feelings of moving the limb (Jeannerod, 1994). Movement imagery as an internal process can therefore be compared with move- sory cortex (S1) was significantly activated only during EM. Ipsilateral cerebellar activation was decreased during IM compared to EM. In the cerebellum, IM and EM differed in their foci of maximal activation: Highest ipsilateral activation of the cerebellum was observed in the anterior lobe (Larsell lobule H IV) during EM, whereas a lower maximum was found about 2-cm dorsolateral (Larsell lobule H VII) during IM. The prefrontal and parietal regions revealed no significant changes during both conditions. The results of cortical activity support the hypothesis that motor imagery and motor performance possess similar neural substrates. The differential activation in the cerebellum during EM and IM is in accordance with the assumption that the posterior cerebellum is involved in the inhibition of movement execution during imagination. ment preparation, two processes that might be functionally equivalent (Jeannerod, 1994). The role of primary and secondary motor areas in movement preparation has been discussed for a long time. Up to a decade ago, movement preparation was thought to be related solely to activity in the prefrontal cortex and the presupplemental motor area (pre-SMA) (Freund, 1996). The Bereitschaftspotential, which can be measured 800 msec before movement onset, was thought to originate in this area. In movement imagery research most of the authors reported increased activation of SMA during imagined movements (IM) compared to executed movements (EM) (cf. Roland et al., 1980). Recently, the sole contribution of SMA activity to the Bereitschaftspotential has been questioned (e.g., Boetzel et al., 1993). The activity that precedes motor action might also be generated in the primary motor cortex (M1) or the cerebellum. Thus, the role of M1 shifted from a mere executional part of the motor system to an area that may also contain more 
elaborate motor functions. If M1 were only an executional part of the motor system, no activity would be expected during the imagination of movements, or if so, this should be due to an artifact of muscle activation during imagined movement. James (1890) and Jacobsen (1930) described that the mental image of a movement is always followed by discharges of its target muscles. Thus, the role of M1 in movement preparation can only be addressed if imagined movement is kept free of muscular discharge.

Using functional magnetic resonance imaging (fMRI), several recent studies reported M1 activation during IM (Leonardo et al., 1995; Porro et al., 1996; Sabbah et al., 1995; Roth et al., 1996) but did not control for possible muscle discharges during IM of a sequential finger-tothumb opposition task. Studies using electrophysiological measurements (Beisteiner, Höllinger, Lindinger, Lang, \& Berthoz, 1995; Naito and Matsumara, 1994) or transcranial magnetic stimulation techniques (Pascual Leone et al., 1995) and controlled EMG-activity during imagery assessment also support the involvement of the contralateral M1 in IM.

In contrast, positron emission tomographic (PET) studies did not report significant activation in the primary motor cortex during IM (e.g., Decety et al., 1994; Roland et al., 1980). However, the low spatial resolution in PET studies makes it difficult, if not impossible, to differentiate between activation in M1 and S1 (as noted by Porro et al., 1996). The lack of reafferent feedback during IM from S1 (Porro et al., 1996) and lower IM-related activation in M1 may lead to the erroneous conclusion that there is a lack of significant activation in the sensorimotor cortex (precentral and postcentral gyrus) during IM. In addition, the type of imagery is important. If subjects are not trained to the kinesthetic imagery, they may visualize hand movements that are likely to involve the occipital cortex but not M1.

Based on these theoretical assumptions and empirical studies, an fMRI investigation of motor imagery was planned. The study had two aims, the first to study activation in the primary and secondary motor areas during EM and IM of the dominant and the nondominant hand using a maximally controlled setting. To exclude frequency-dependent artifacts (Schlaug et al., 1996), the movements were acoustically metronomepaced at a rate of one per second. Furthermore, IM was trained under electromyographic (EMG) control by visual EMG feedback prior to the fMRI measurement. Subjects were instructed and reinforced to create a kinesthetic image of the motor movement without discharges of the muscles or involvement of visual imagery. In addition, subjects were preselected based on their imagery ability.

The second aim of the study was to use the high spatial resolution of fMRI and the measurement of a whole brain dataset of the cerebrum and the cerebellum to localize activation maps during EM and IM and to determine to what extent they share common neural mechanisms.

\section{RESULTS}

The EMG data of all subjects who participated in the experiment are shown in Figure 1. The initial EMG value of the musculus flexor digitorum superficialis of the right hand involved in imagery was $12.39 \mu \mathrm{V}$ ( $S D=$ 2.94); this value dropped to $1.45 \mu \mathrm{V}(S D=0.11)$ at the end of the training, a value that was not significantly different from the resting baseline $(M=1.83 \mu \mathrm{V} ; S D=$ 0.20 ), although all subjects showed high levels of imagery vividness ( $\geq 4$ of a 6 -point scale).

In Figure 2, the typical activation during right-hand EM and IM of one subject is shown. During EM (Figure 2, top row), strong activation in the contralateral M1 and S1, less activation in the SMA of both hemispheres, and strong activation in the ipsilateral anterior cerebellar lobe was observed. Compared to EM, activation during IM was stronger in SMA, lower in M1, and particularly low in S1 and the anterior cerebellum. These individual data are mirrored quite well in the counted pixels of the selected regions averaged over all subjects (Figures 2 and 3).

The percentage of activated pixels above a $t$ value of $2.45(p<0.01)$ is shown in Table $1 \mathrm{~A}$ and Figure $3 \mathrm{~A}$ for the cortical regions and in Table $1 \mathrm{~B}$ and Figure $3 \mathrm{~B}$ for the cerebellar regions.

A multivariate analysis of variance (MANOVA) was performed on all selected cortical and cerebellar regions using three factors: left versus right hemisphere (laterality), hand used for EM and IM (hand), and type of movement (executed versus imagined: EM/IM). Significant MANOVA results were followed by paired $t$ tests to further evaluate the differences in activation dependent on the condition. To determine the significance of regional activation during the different tasks, the cortical regions were also compared to a temporal reference region.

\section{Cortical Regions}

M1

MANOVA revealed the following main effects: Significant lateralization $(F=17.38 ; p<0.005)$, significant hand dependency $(F=6.47 ; p<0.05)$, but no significant differences for EM/IM. A significant interaction was found for laterality and EM/IM $(F=16.63 ; p<0.005)$. EM showed higher lateralization to the contralateral hemisphere than IM. The interaction of all three factors showed a trend toward significance $(F=4.83 ; p=0.06)$, indicating differences in ipsilateral and contralateral primary motor cortex activation dependent on the used hand and the task (EM versus IM). 
Figure 1. EMG average amplitude data reflecting discharges of the musculus flexor digitorum superficialis of all subjects during rest (baseline, left black bar) and executed move ment of the left hand (EM, left gray bar). On the right side baseline (black bar) and the last three training trials of imagined movement (IM, gray bar) are depicted.

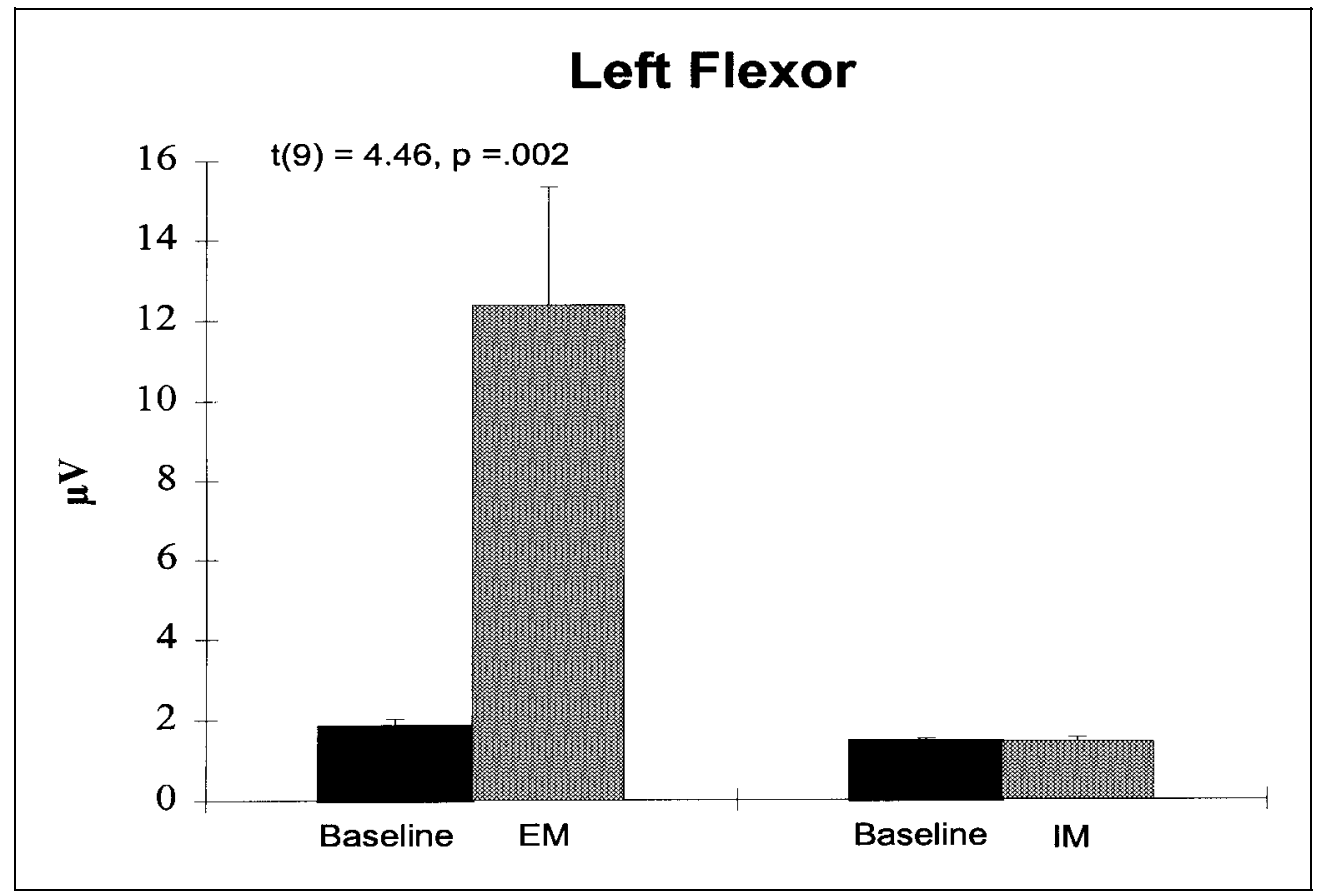

\section{Somatosensory Cortex (S1)}

In S1, lateralization $(F=9.91 ; p<0.05)$, hand $(F=5.26$; $p<0.05)$, and EM/IM $(F=13.97 ; p=0.005)$ were significant. A significant interaction was also seen for laterality and EM/IM $(F=10.69 ; p=0.01)$.

Post hoc comparisons revealed that the lateralization effect to the contralateral M1 and S1 during EM was less pronounced for the left than for the dominant right-hand movement $(t$-test right hand; contralateral against ipsilateral ROI: M1: $t=7.4, p<0.001 ; \mathrm{S} 1: t=3.0, p<0.05$; left hand: M1: $t=1.2, n . s . ; \mathrm{S} 1: t=1.9, n . s$.$) . During right-hand$ IM, the contralateral M1 showed significantly higher activation compared to the temporal reference region (M1: $t=2.2 ; p<0.05$ ), whereas $\mathrm{S} 1$ failed to show significantly higher activation (S1: $t=1.5 ;$ n.s.). Contralateral M1 showed a reduction in activation of about $50 \%$ during imagined as compared to actual movement. Contralateral S1 had about $70 \%$ less activation during IM compared to EM (contralateral S1: right hand: $t=4.1 ; p<0.05$; left hand: $t=2.6 ; p<0.05$ ). Furthermore, ipsilateral activation in M1 was not significantly different between EM and IM (right hand: $t=0.2 ; n . s$.; left hand: $t=0.4 ; n . s$.).

\section{$S M A$}

None of the main effects were significant. Over all subjects, SMA showed no higher activation during IM compared to EM, and there was no lateralization effect.

\section{Premotor Cortex (PMC)}

In PMC, no significant main or interaction effects could be detected except for a significant hand effect $(F=$
5.11; $p=0.05) . T$ tests showed that the right hand produced stronger activation in PMC than the left hand.

The frontal lobe, the parietal lobe, and the cingulate gyrus revealed no significant effects. Table 1A and Figure $3 \mathrm{~A}$ show the regional activation in detail.

\section{Cerebellar Regions}

In the anterior cerebellar lobes, lateralization $(F=62.28$; $p<0.001)$ and EM/IM $(F=19.69 ; p<0.005)$ were significant. A significant interaction was observed for lateralization and EM/IM $(F=73.54 ; p<0.001)$. In the lower cerebellum and the vermis, MANOVA yielded no significant results. $T$ tests showed highly significant lateralization during EM to the ipsilateral anterior hemisphere (right hand: $t=6.5 ; p<0.001$; left hand: $t=6.3$; $p<0.001$ ), which was lower during IM (right hand: $t=$ 3.0; $p<0.05$; left hand: $t=1.3$; n.s.). During EM the ipsilateral posterior lobe was about $60 \%$ less activated than the anterior lobe (right hand: $t=4.1 ; p<0.005$; left hand: $t=5.5 ; p<0.001$; see Table $1 \mathrm{~B}$ and Figure 3B). The cerebellar vermis showed no significant differences when the activation in the anterior part was tested against the activation in the posterior part. During IM, activation in the ipsilateral anterior lobe was reduced to $30 \%$ of EM activation (right hand: $t=3.7, p<0.005$; left hand: $t=6.9, p<0.0005$; see Figure 3B, Table 1B). The activation in the ipsilateral posterior cerebellar lobes revealed no significant differences when IM was compared to EM for the right hand $(t=0.2$, n.s.) but significant differences for the left hand $(t=2.6 ; p<0.05)$.

When circumscribed activation maxima were analyzed, the following results emerged: For EM, the location 
Figure 2. Top row: Activation in one subject during EM of the right hand in five slices. Bottom row: Activation of the same subject during IM right for the same slices.

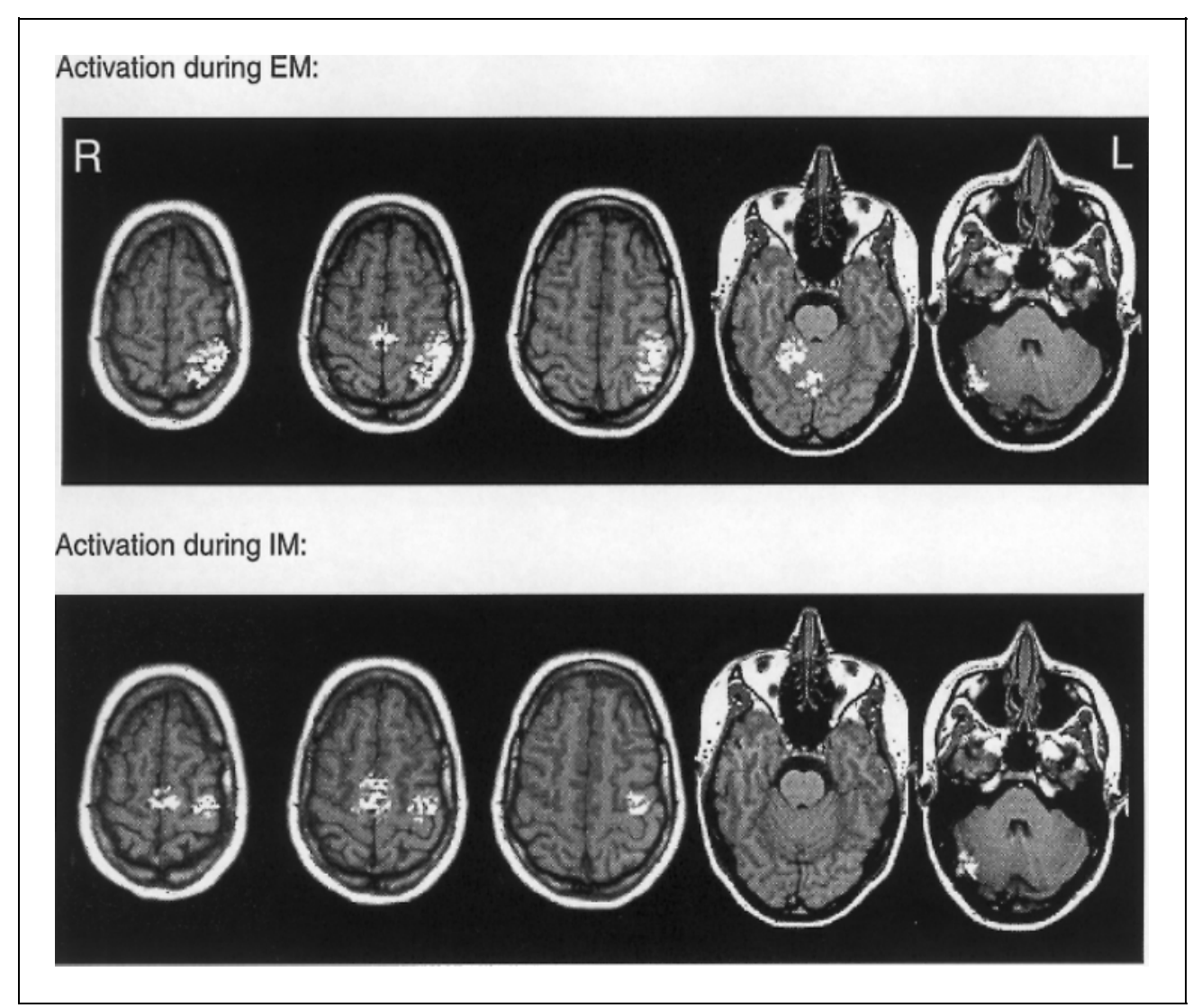

of the maximum was found in the Larsell lobule $\mathrm{H}$ IV and for IM, in the Larsell lobule $\mathrm{H}$ VII. The activation pattern during IM was compared to that during EM by selection of the same cutoff threshold for both conditions (see Figure 4). EM of both hands showed no activation in the lateral part of the cerebellar hemisphere (Larsell lobule H VII) where the activation maxima during IM were localized. The maximum of cerebellar activity during IM was about $1 \mathrm{~cm}$ more lateral and $0.5 \mathrm{~cm}$ more dorsocaudal than for EM.

\section{DISCUSSION}

Our study shows that the same cortical regions that subserve the execution of movement are active during motor imagery. Whereas SMA and PMC were equally activated during both actual and imagined movement, M1 and especially S1 showed significantly less activation during IM. These results are in accordance with previous findings (e.g. Leonardo et al., 1995; Porro et al., 1996; Roth et al., 1996) and add further support to the notion that motor imagery is functionally and anatomically related to motor performance. The cerebral activation observed in our study was not related to residual motor activity because subjects were explicitly trained in a simulated magnetic resonance (MR) environment to avoid all motor activation during imagined movement. The same amount of activation in the SMA and the PMC during EM and IM supports the hypothesis of functional equivalence of motor imagery and motor preparation postulated by Jeannerod (1994). The SMA has integrative functions for the planning of complex motor tasks. As confirmed by the data of this study, the SMA is involved in the processing of imagery of movements as well as the execution of simple hand movements. Our fMRI data are in agreement with findings from electrophysiology (Beisteiner et al., 1995; Goldberg, 1985) and other fMRI studies (e.g., Stephan et al., 1995). Some authors (Decety et al., 1994; Roland et al., 1980) did not report any significant SMA or M1 activation during IM. This is probably due to different imagination tasks. For instance, Decety used a task where the subjects observed passive movements of an alien hand. Because this is not a kinesthetic imagination task, the differences in activation compared to motor imagination can be explained by these methodological differences. SMA activation is equivalent to or even more pronounced than EM in all studies dealing with kinesthetic motor imagery (e.g., Porro et al., 1996). The significant activation in M1 indicates that the primary motor cortex contributes to movement preparation and that its function is not restricted to the mere execution of movements. In our study the ipsilateral and contralateral M1 activation during IM was comparable to the activation during EM, whereas the activation in S1 was significantly reduced during IM compared to EM. Furthermore, the activation in S1 during IM was not significantly stronger than that of a temporal cortical reference region. Sensory afferents seem not to contrib- 
Figure 3. Activated pixels in percentage of total number of pixels in cortical ROIs for all significantly activated pixels $(p<0.01)$ of 10 subjects. White colored bars: executed movement; gray colored bars: imagined movement; bars without lines: right hand movement; striped bars: left hand movement. SEM indicated in error lines on top of bars. Asterisks denote significant differences in activation $\left({ }^{*} p<0.05 ;{ }^{* *} p<0.01\right)$. (A) Cortical activation in M1, S1, SMA, PMC, and CG. (B) Cerebellar activation in the anterior and posterior hemisphere.

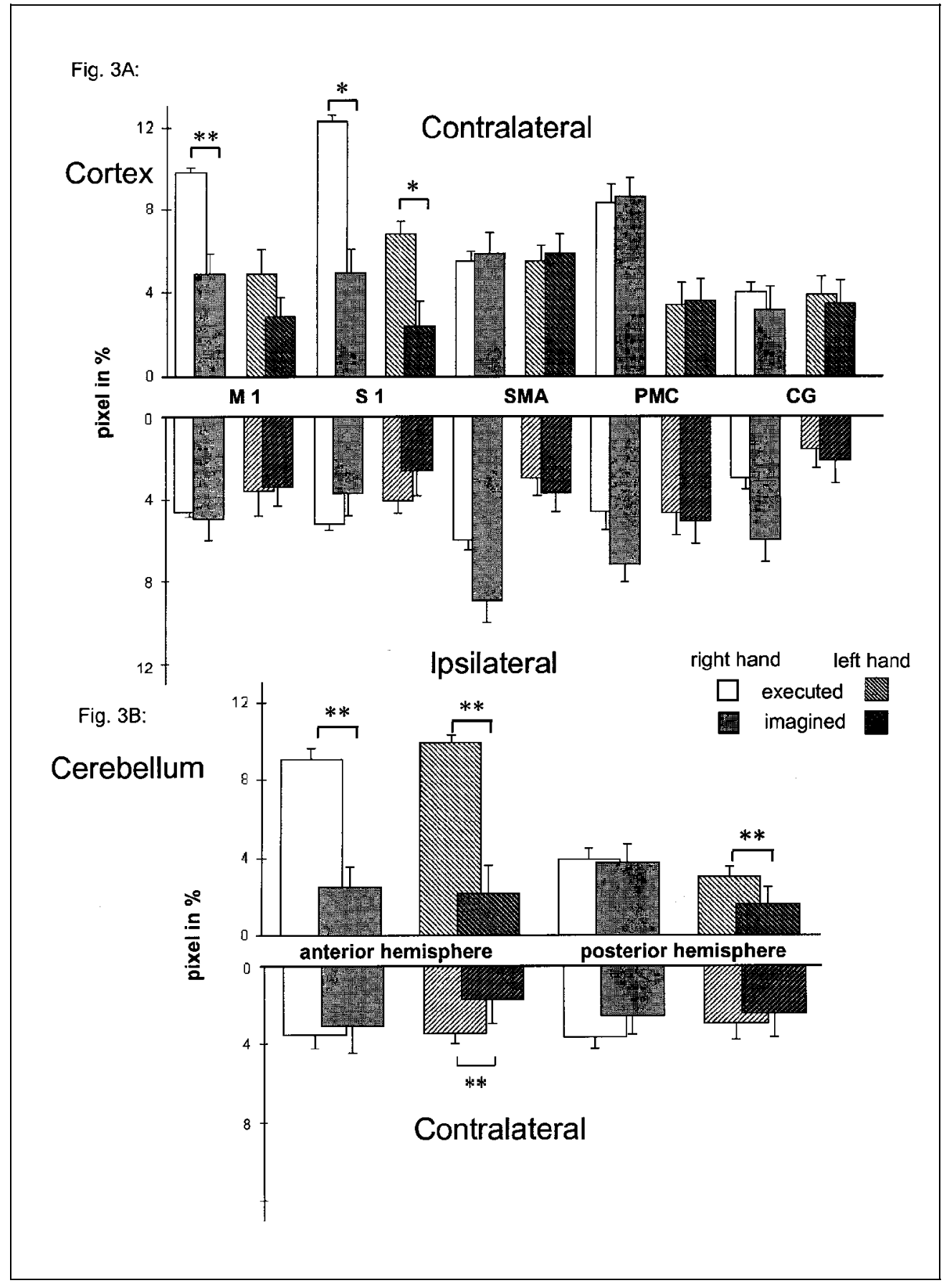

ute significantly to motor imagery. A recent MEG study compared activation in M1 and S1 during electric median nerve stimulation with imagined sensory stimulation (Schnitzler, Salenius, Salmelin, Jousmaki, \& Hari, 1997). Here, M1 but not S1 showed significant activation during IM. The fact that S1 did not produce significant activation during IM supports the assumption that methods with lower spatial resolution may not discriminate activation in the precentral and the postcentral gyrus.

The dominant hand shows high lateralization to the contralateral M1 and S1, whereas for the nondominant left-hand lateralization is less pronounced (Kim, Ashe, \&
Hendrich, 1993). Imagination of movement failed to show significant lateralization in M1 for the dominant hand. This may be due to a higher complexity of the imagined task. During increased complexity of a motor task, higher ipsilateral activation in M1 was reported (e.g., Mattay et al., 1998).

In our study, motor imagery as compared to EM did not show higher parietal activity as reported for the left parietal lobe during visual imaginary (Farah, 1995). For the right-hand movement, the left parietal lobe was significantly more activated during EM than during IM. These differences in parietal activation during imagery 
Table 1. Number of activated pixels with a $t$ value exceeding $2.45(p<0.01)$ in percentage of the total number of pixels in the selected anatomical regions of 10 subjects. Table 1A shows the results for the cortical, and Table $1 \mathrm{~B}$ shows the results for the cerebellar regions.

\begin{tabular}{|c|c|c|c|c|c|c|c|c|c|c|c|c|c|c|c|}
\hline \multirow{2}{*}{ A Region } & \multicolumn{2}{|c|}{ M1 } & \multicolumn{2}{|c|}{$S 1$} & \multicolumn{2}{|c|}{$S M A$} & \multicolumn{2}{|c|}{$P M C$} & \multicolumn{2}{|c|}{ Cing. Gyr } & \multicolumn{2}{|c|}{ Prefront. } & \multicolumn{2}{|c|}{ Parietal } & \multirow{2}{*}{$\begin{array}{c}\text { Reference } \\
\text { Temp. } \\
\text { Gyrus }\end{array}$} \\
\hline & Contra & $I p s i$ & Contra & Ipsi & Contra & $I p s i$ & Contra & $I p s i$ & Contra & $I p s i$ & Contra & $I p s i$ & Contra & $I p s i$ & \\
\hline EM right hand & 9.8 & 4.6 & 12.3 & 5.2 & 5.5 & 6.0 & 8.3 & 4.6 & 4.0 & 3.0 & 3.3 & 3.5 & 4.6 & 3.7 & 2.2 \\
\hline IM right hand & 4.9 & 5.0 & 5.0 & 3.7 & 5.9 & 9.0 & 8.6 & 7.2 & 3.2 & 6.0 & 4.0 & 5.2 & 2.8 & 3.9 & 2.4 \\
\hline EM left hand & 4.9 & 3.6 & 6.8 & 4.1 & 5.5 & 3.0 & 3.4 & 4.7 & 3.9 & 1.6 & 3.4 & 2.8 & 2.9 & 3.0 & 1.4 \\
\hline IM left hand & 2.9 & 3.4 & 2.4 & 2.6 & 5.9 & 3.7 & 3.6 & 5.1 & 3.5 & 2.1 & 3.1 & 3.0 & 2.5 & 2.0 & 1.5 \\
\hline
\end{tabular}

\begin{tabular}{|c|c|c|c|c|c|c|}
\hline \multirow[b]{2}{*}{ Region } & \multicolumn{2}{|c|}{ Anterior Lobe } & \multicolumn{2}{|c|}{ Posterior Lobe } & \multicolumn{2}{|c|}{ Vermis } \\
\hline & Contra & Ipsi & Contra & $I p s i$ & Anterior & Posterior \\
\hline EM right hand & 3.6 & 9.0 & 2.6 & 3.9 & 6.4 & 4.6 \\
\hline IM right hand & 3.1 & 2.5 & 2.6 & 3.7 & 3.4 & 3.8 \\
\hline EM left hand & 3.5 & 9.9 & 3.0 & 3.0 & 3.8 & 4.3 \\
\hline IM left hand & 1.7 & 2.2 & 2.5 & 1.6 & 1.7 & 2.1 \\
\hline
\end{tabular}

may be due to the different type of image modality. In our study a very simple hand movement was performed, during which no parietal activation is expected. High activity of the parietal cortex was observed only in motor tasks, which include complex movements coordinated in space, such as graphomotor control (Seitz et al., 1997).

The present study indicates that the cerebellum is less activated during imagery of simple hand movements compared to actual movement. This finding is in agreement with previous PET and single photon emission computerized tomography (SPECT) studies showing decreased blood flow within the cerebellum during motor imagery compared to motor execution (Decety et al., 1994, Ryding, Decety, Sjöholm, Stenberg \& Ingvar, 1993). The reduced activity, especially of the anterior cerebellar lobe during motor imagery, might be related to a lack of afferent information during the task. This assumption is in accordance with findings of Gao et al. (1996), indicating that the Larsell lobule $\mathrm{H}$ IV of the ipsilateral anterior cerebellar hemisphere is involved in sensory information processing. Movements of the hand activate the same location (Nitschke, Kleinschmidt, Wessel, \& Frahm, 1996, Britsch et al., 1996). Significant reduction of activation in S1 during IM compared to EM was also reported for MEG data by Schnitzler et al. (1997).

As shown in Figure 4, no activation in the Larsell lobule H VII can be observed during EM; the activation pattern during EM is localized strictly to the Larsell lobule H IV to V, to the vermis, and to the medial part of the posterior hemisphere. Therefore different cerebellar neurons seem to be activated during IM than during EM.
An inhibitory activation of the lateral cerebellar hemisphere with respect to the execution of movement might underlie the observed activation maximum in the Larsell lobule H VII during IM. Sakai et al. (1997) previously reported an activation maximum during no-go motor tasks in the Larsell lobule H VII. These neuronal groups may also be responsible for the circumscribed activation during the imagination task. The trained subjects had to avoid any execution of the movement. This task might correspond to a no-go task. The cerebellar activation that is described here may offer a missing link in the model of Jeannerod (1994), who explained the differences between imagination of movement and movement preparation by the fact that the content of motor images can be accessed consciously, whereas movement preparation remains nonconscious until the movement is blocked. Our data suggest that this inhibitory process may be located in the cerebellum, specifically in the Larsell lobule HVII ipsilateral to the movement.

The activation maxima in the ipsilateral Larsell lobule HVII during IM were probably not caused by a higher attentional demand: The activation in this area was not lateralized to the left hemisphere as reported for attentional activation in the lateral cerebellum (Allen, Buxton, Wong, \& Courchesne, 1997). We assume further that the activation in the Larsell lobule H VII is not due to temporal aspects of imagination of the movement, although the activation maximum in the Larsell lobule $\mathrm{H}$ VII is consistent with the localization of timing processes in the lateral cerebellum (Leiner, Leiner, \& Dow, 1995; Strick, Hoover, \& Mushiake, 1993). However, the tempo- 
Figure 4. Activation during different types of movement in the normalized and overlaid cerebella of 10 subjects. Note that the cutoff level is the same during EM and IM. In each row the left side depicts the coronal view, the middle, the axial view, and the right side, the sagittal view. L indicates the left side, and $\mathrm{R}$ indicates the right side of the dataset. (A) Cerebellar activation during right-hand movement. Top row: Activation during EM. The activation maximum lies in the ipsilateral anterior hemisphere (Larsell lobule H IV-V). Despite of the low cutoff level, there is no activation in the lateral part of the hemisphere (Larsell lobule H VII). Bottom row: Activation during IM of the right hand. The activation maxima lies in the ipsilateral posterior hemisphere (Larsell lobule H VII) $2.2 \mathrm{~cm}$ more lateral and $1 \mathrm{~cm}$ more dorsal than the activation maxima during EM (B) Cerebellar activation during left-hand movement. Top row: Activation maximum during EM is located in the ipsilateral anterior hemisphere (Larsell lobule $\mathrm{H} \mathrm{IV}-\mathrm{V}$ ) but $5 \mathrm{~mm}$ more dorsal than right-hand EM. The activation pattern during EM showed no activation in the lateral part of the cerebellar hemisphere in the Larsell lobule H VII where the activation maxima during IM is localized. Bottom row: The activation maxima during IM lies in the ipsilateral posterior hemisphere (Larsell lobule $\mathrm{H}$ VII) $2.2 \mathrm{~cm}$ more lateral and $1 \mathrm{~cm}$ more dorsal than activation maxima during EM and also in the contralateral hemisphere at the same location as during IM of the dominant hand. The activation maximum during left-hand IM lies $7 \mathrm{~mm}$ more dorsal than the ipsilateral activation maximum during right-hand IM.
Figure 4A:

\section{EM right hand:}

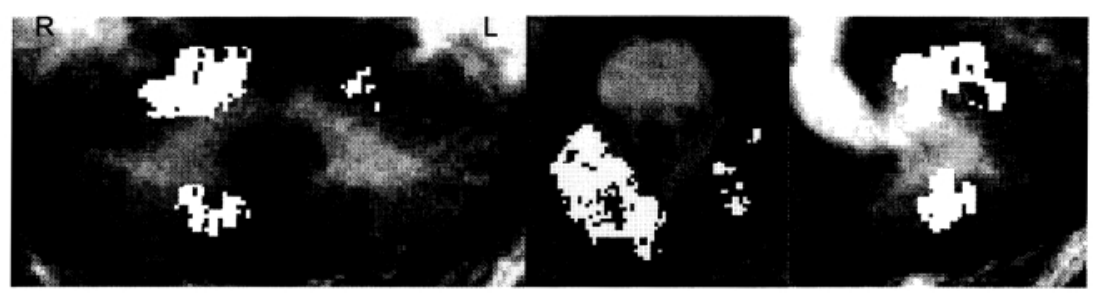

IM right hand:

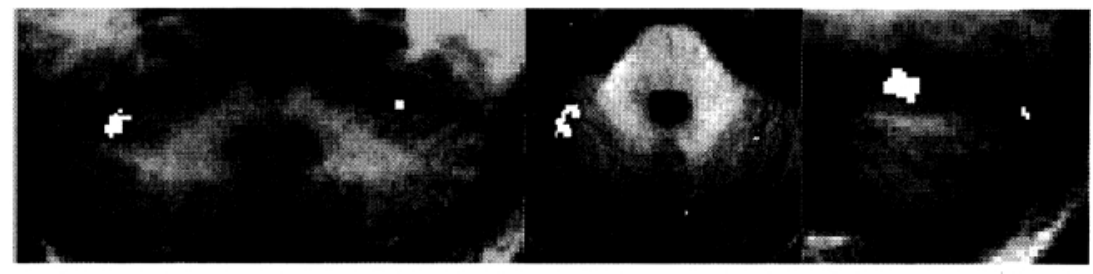

Figure 4B:

\section{EM left hand:}

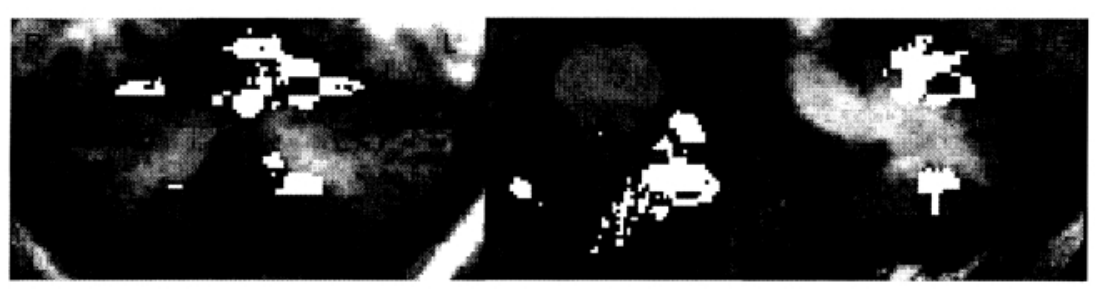

IM left hand:

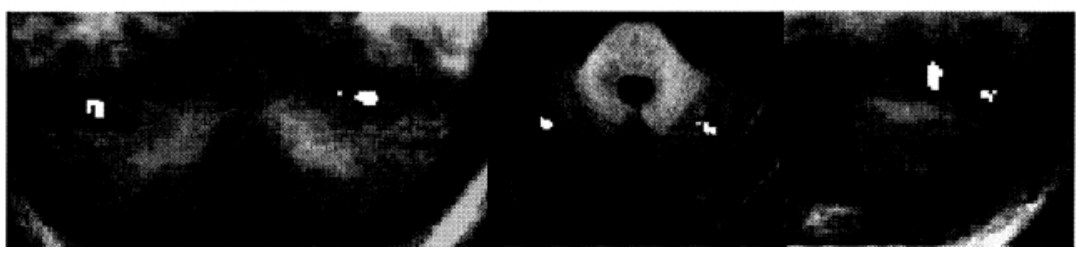

ral aspects of movement should be the same during IM and EM (c.f. Decety, Jeannerod, \& Prablanc, 1989). No additional temporal sequencing of the imagined movement was necessary compared to EM because both movements were triggered by the same metronome sounds.
The primary motor and somatosensory cortex and the anterior cerebellum showed significant differences in activation between the imagination and execution task of the dominant hand. No significant differences were seen between these tasks in the activation of the SMA, the PMC, and the posterior cerebellar lobes. When acti- 
vation in M1 and S1 of the contralateral cortex and of the ipsilateral anterior cerebellar lobe were compared between EM and IM, similar proportions of activation emerged. The amount of activation that was observed during EM was reduced during IM to about 50\% in M1, to about $30 \%$ in $\mathrm{S} 1$, and to about $30 \%$ in the anterior cerebellar lobe. We assume that the decrease of activation in the anterior cerebellum is related to the lack of afferent information during IM.

The activation in the SMA, the premotor cortex, and the posterior ipsilateral cerebellar lobe during movement of the dominant hand was not reduced if EM was compared to IM. These two phenomena could be explained by neuroanatomic pathways between the different cerebellar lobes and the primary and secondary motor areas. Anatomically, the cerebellum receives sensory information via the spino-cerebellar tract. Information about cortical control of movement is provided by the cortico-pontino-cerebellar tract. Information from the anterior cerebellum passes via the dorsal nucleus dentatus to the contralateral M1 and S1. The ventral part of the nucleus dentatus is connected primarily to the dorso-lateral prefrontal parts of the cortex (Middleton \& Strick, 1994). This tract closely links the upper part of the posterior cerebellum to the SMA and the premotor cortex. Along this pathway, aspects of movement coordination and also inhibition of movement execution may be connected between the SMA and the lateral cerebellar hemisphere (Rao et al., 1997).

A primary result of this study is the high concordance of EM and IM with respect to activation in the secondary and primary motor areas, supporting the notion that motor preparation and imagery share common neural substrates (Jeannerod, 1994). This was found with primarily kinesthetic imagery with concurrent control of EMG activity and imagery ability as well as the pace of the movement. The cerebellar data also suggest that motor preparation may have to be inhibited when motor imagery is performed.

\section{METHODS}

\section{Subjects}

Ten right-handed volunteers (five male and five female, 19 to 40 years) with no neurological complications were examined.

\section{Training}

Subjects first had to make a fist and then to imagine making a fist in a sequence of one movement per second (metronome paced). First right-hand and then left-hand movements were trained. Surface EMG were recorded over the target muscle (musculus flexor digitorum superficialis of the forearm) during training. The task for the subjects was to achieve the highest possible intensity of subjective ratings of vividness of the imagined movements [verbal report using a seven-point scale ranging from 0 (no image present at all) to 6 (perfectly clear and vivid image)] with complete avoidance of any EMG amplitude higher than during the rest condition (visual feedback). The training had a total duration of 60 to 90 min using simulated fMRI conditions (supine position, machine noise on tape, metronome pacing) and was terminated when the subjects' report of vividness reached a score of 4 and the EMG level during imagined movements no longer exceeded the baseline level (see Figure 1).

\section{Measurement}

The actual measurement in the scanner was performed within $60 \mathrm{~min}$ after the training session under the same procedure as during training. Four conditions were examined in the following order: (1) actual movement with the right hand, (2) imagined movement with the right hand, (3) actual movement with the left hand, and (4) imagined movement with the left hand. The whole duration of the measurement with anatomical data acquisition was about $90 \mathrm{~min}$ including about $10 \mathrm{~min}$ for each functional measurement. Each condition consisted of 1 min of action (60 executed or imagined movements), which was preceded by a $1-$ min rest period. These activation and resting conditions were repeated four times each. Subjective ratings of the vividness of the imagined movements were obtained by verbal report during the subsequent rest periods. Movements were paced acoustically by a metronome with a frequency of one per second.

\section{Data Acquisition}

Whole brain fMRI data acquisition was performed with a commercial 1.5 Tesla whole body tomograph (Siemens Vision) using a multislice echo planar imaging sequence (EPI) with 27 axial slices [4-mm slice thickness, 1-mm gap, $96 \times 128$ matrix, field of view (FOV) $260 \times 162 \mathrm{~mm}$, echo time (TE) $46 \mathrm{msec}$, flip angle $\alpha 90^{\circ}$, acquisition time $4 \mathrm{sec}$. During each of the four repetitions of the experimental conditions, 24 sets of 27 slices were taken during the resting baseline and the same number of sets were taken during the task periods, yielding 1296 images per condition (measurement interval, $4 \mathrm{sec}$; repetition interval, 8 sec). The subjects were lying supine with their eyes closed in the scanner with a secure fixation of the head and the proximal limb to minimize involuntary movements. The remaining head movements were evaluated by a displacement analysis. Movements were detected by calculating correlation coefficients of each single dataset with a reference dataset after a shift in a chosen direction (Klose, Grodd, Skalej, Kolb, \& Naegele, 1997). Movements along all three spatial directions were examined. Images with movements of more than 0.3 pixels were 
excluded from further analysis. In addition, a corresponding set of T1-weighted spin echo images (repetition time, $500 \mathrm{msec}$, TE $12 \mathrm{msec}$ with identical slice positions, slice thickness, and FOV as for the EPI dataset) were acquired for the definition of anatomical regions as well as a 3D dataset (T1-weighted FLASH images with 128 sagittal slices) for the visualization of normalized data.

\section{Data Analysis}

For both EM and IM, Talairach normalization of the averaged data revealed marginal spatial resolution with no clear differentiation of the precentral and postcentral gyrus (see Figure 5, top row). Thus, further statistical analysis of activated pixels in circumscribed anatomical regions was not possible based on this averaged dataset. Therefore, we defined anatomically selected ROIs (for four selected slices see Figure 5, bottom row) in each of the 10 datasets and analyzed the activation within these regions. ROIs were defined in each of the 27 anatomical T1-weighted slices [M1, S1, SMA, PMC, both frontal and parietal lobes, the cingulate gyrus (CG), the right temporal superior and medial gyrus as reference region, both cerebellar hemispheres, and the vermis]. The anatomical landmarks were taken from Mai, Assheuer, \& Paxinos (1997). Statistical evaluation of the fMRI data was performed by calculation of $t$-value maps. The number of pixels above the chosen threshold were counted in the different ROIs. In addition to the ordinarily selected $p$ value of 0.01 as a cutoff limit, the threshold $t$ value was assessed by comparing the ROI with the assumed maximal activation in the contralateral M1 for EM and the assumed lowest activation in the cortex of the medial superior temporal gyrus and in the right hemisphere (Klose, Lotze, \& Grodd, 1998). A $t$ value of $2.45(p<0.01)$ lay in the medial part of the receiver operating characteristics (ROC) curve, which revealed a high difference between motor areas and control regions in all conditions and was therefore selected for further data analysis. The number of activated pixels was calculated as the percentage of the total number of pixels within each ROI. MANOVA was used to evaluate differences of the used hand, hemisphere lateralization, and differences between EM and IM for each ROI, and $F$ values were calculated with 1 degree of freedom. Two-tailed $t$ tests for the comparison of different ROIs were also calculated by using the percentage of activated pixels within each region compared to total number of pixels per region.

\section{Data Display}

Counting activated pixels in different ROIs is advantageous for statistical comparisons of activation; however, this method shows disadvantages in the description of circumscribed activation maxima. For individual data visualization, a spatial filtering of the parameter maps was performed (Figure 2). The matching of the cortical data was performed with a Talairach alignment of the 3-D anatomical and functional dataset (see Figure 5A) using the AFNI program (Cox, 1996). For the cerebellum (Fig ure 4), a linear transformation algorithm was used with reference points at the fourth ventricle and the cranial, caudal, anterior, posterior, and the lateral boarders of the cerebellar hemispheres because the Talairach transformation revealed an unsatisfactory superposition (reference points near the basal ganglia). This method is described elsewhere in detail (Huelsmann, Erb, Lotze, \& Grodd, 1998).
Figure 5. Top row: Talairach normalized data of all 10 subjects are shown for four slices during right-hand movement. Bottom row: Anatomically selected ROIs on T1-weighted images of one subject are shown for 4 of 27 slices (PMC: premotor cortex; SMA: supplementary motor area; M1: precentral gyrus, primary motor cortex; $\mathrm{S} 1$ : postcentral gyrus, primary somatosensory cortex; CG: cingulate gyrus; AC: anterior cerebellar hemisphere).

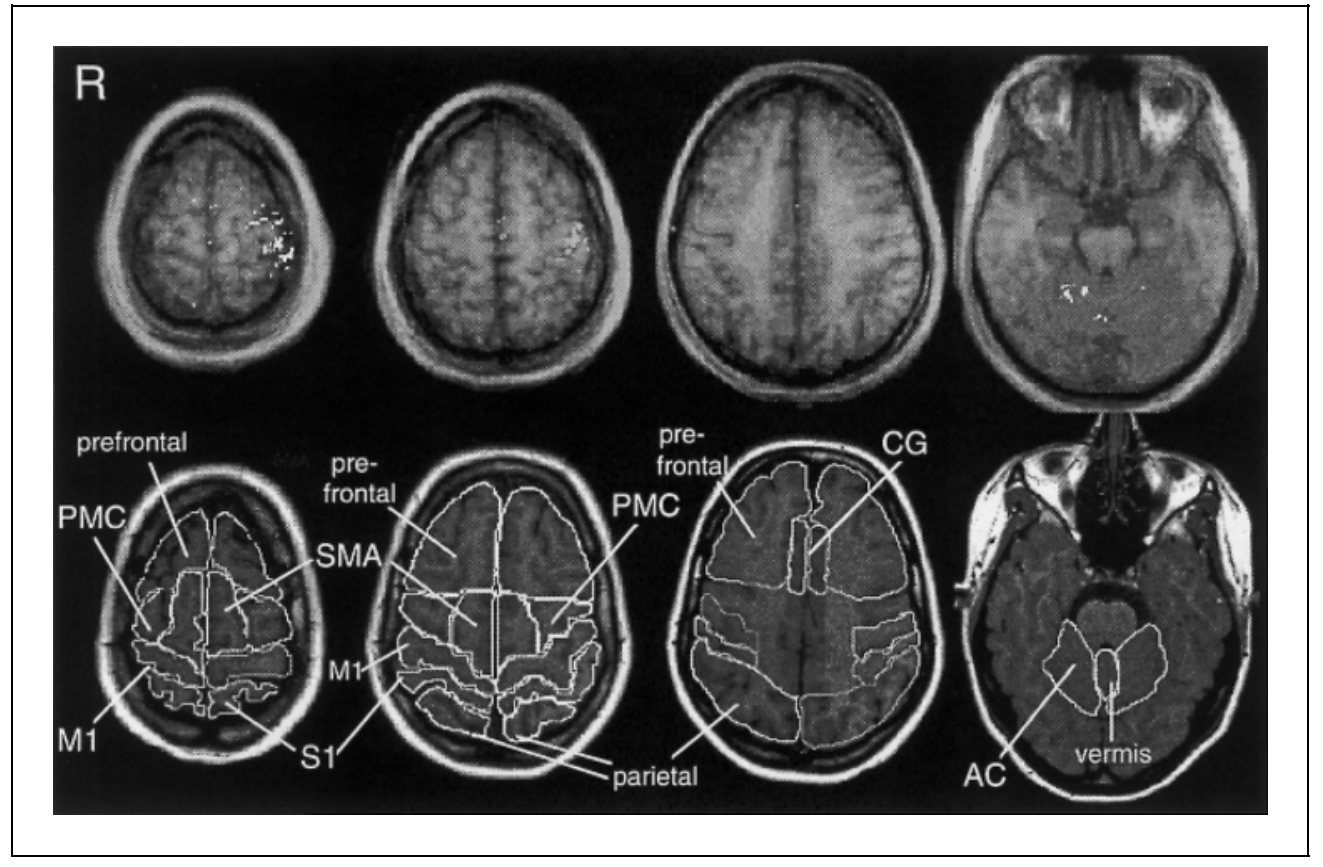




\section{Acknowledgments}

We thank Mrs. Ingrid Schneider for technical assistance in data measurement. Furthermore, Dr. Bärbel Knost helped generously in the evaluation of the EMG data. This study was supported by the Federal Ministry for Education, Science and Technology, by the Deutsche Forschungsgemeinschaft (DFG, Bi 195/24), the Fortüne Program (\# 249) of the Medical Faculty, University of Tübingen and the "Volkswagen-Stiftung."

Reprint requests should be sent to Martin Lotze, Institute for Medical Psychology, Gartenstrasse 29, D-72074, Tübingen, Germany, or via e-mail: martin.lotze@uni-tuebingen.de.

\section{REFERENCES}

Allen, G., Buxton, R. B., Wong, E. C., \& Courchesne, E. (1997). Attentional activation of the cerebellum independent of motor involvement. Nature, 275, 1940-1941.

Beisteiner, R., Höllinger, P., Lindinger, G., Lang, W., \& Berthoz, A. (1995). Mental representations of movements: Brain potentials associated with imagination of hand movements. Electroencephalography and Clinical Neurophysiology, 96, 183-193.

Boetzel, K., Plendl, H., Paulus, W., \& Scherg, M. (1993). Bereitschaftspotential: Is there a contribution of the supplementary motor area? Electroencephalography and Clinical Neurophysiology, 89, 187-196.

Britsch, P., Grodd, W., Klose, U., Ackermann, H., Kardatzki, B., \& Voigt, K. (1996). Functional MRI of the cerebellum during voluntary hand and foot movement with multislice EPI. Neuroimage, 3, S378.

Cox, R. W. (1996). AFNI: Software for analysis and visualization of functional MR. Neuroimages, Computing in Biomedical Research, 29, 162-173.

Decety, J., Jeannerod, M., \& Prablanc, C. (1989). The timing of mentally represented actions. Behavioral Brain Research, 34, 35-42.

Decety, J., Perani, D., Jeannerod, M., Bettinardi, V., Tadary, B., Woods, R., Maziotta, J. C., \& Fazio, F. (1994). Mapping motor representations with PET. Nature, 371, 600602.

Farah, M. J. (1995). The neural basis of mental imagery. In M. S. Gazzaniga (Ed.), The cognitive neurosciences (pp. 963-975). Cambridge, MA: MIT Press.

Freund, H. J. (1996). Historical overview. In H. O. Lüders (Ed.), Supplementary sensorimotor area: Advances in neurology (pp. 17-27). Philadelphia: Lippincott-Raven.

Gao, J-H., Parsons, L. M., Bower, J. M., Xiong, J., Li, J., \& Fox, P. T. (1996). Cerebellum implicated in sensory acquisition and discrimination rather than motor control. Science, 272, 545-547.

Goldberg, G. (1985). Supplementary motor area structure and function: Review and hypothesis. Behavioral Brain Science, 8, 567-616.

Huelsmann, E., Erb, M., Lotze, M., \& Grodd, W. (1998). Mapping of the cerebellum: Interindividual analysis of fMRI activation. Neuroimage, 7, S995.

Jacobson, E. (1931). Electrical measurements of neuromuscular states during mental activities. VIII. Imagination, recollection, and abstract thinking involving the speech musculature. American Journal of Physiology, 97, 200209.

James, W. (1890). Principles of psychology. New York: Macmillan. [New edition: New York: Dover, 1950.]

Jeannerod, M. (1994). The representing brain: Neural corre- lates of motor intention and imagery. Brain and Behavioral Sciences, 17, 187-245.

Kim, S. G., Ashe, J., \& Hendrich, K. (1993). Functional magnetic resonance imaging of motor cortex: Hemispheric asymmetry and handedness. Science, 261, 615-617.

Klose, U., Grodd, W., Skalej, M., Kolb, R., \& Naegele, T. (1997). Evaluation strategies for functional magnetic resonance imaging. In L. Heuser \& M. Oudkerk (Eds.), Advances in MRI (pp. 40-46). Hamburg: Blackwell Science.

Klose, U., Lotze, M., \& Grodd, W. (1998). Comparison of fMRI evaluation parameters utilizing a prior knowledge of expected activation. Neuroimage, 7, S602.

Kosslyn, S. M. (1988). Aspects of a cognitive neuroscience of mental imagery. Science, 240, 1621-1626.

Leiner, H. C., Leiner, A. L., \& Dow, R. S. (1995). The underestimated cerebellum. Human Brain Mapping, 2, 244-254.

Leonardo, M., Fieldman, J., Sadato, N., Campbell, G., Ibanez, V., Cohen, L., Deiber, M. P., Jezzard, P., Pons, T., Turner, R., Le Bihan, D., \& Hallet, M. (1995). A functional resonance imaging study of cortical regions associated with motor task execution and motor ideation in humans. Human Brain Mapping, 3, 83-92.

Mai, J. K., Assheuer, J., \& Paxinos, P. (1997). Atlas of the human brain. San Diego: Academic Press.

Mattay, V. S., Callicott, J. H., Bertolino, A., Santha, A., Van Horn, J. D., Tallent, K. A., Frank, J. A., \& Weinberger, D. R. (1998). Hemispheric control of motor function: A whole brain fMRI study. Neuroimage, 7, S61.

Middleton, F. A., \& Strick, P. L. (1994). Anatomical evidence for cerebellar and basal ganglia involvement in higher cognitive function. Science, 266, 458-461.

Naito, E., \& Matsumara, M. (1994). Movement related slow potentials during motor imagery and motor suppressions in humans. Cognitive Brain Research, 2, 131-137.

Nitschke, M. F., Kleinschmidt, A., Wessel, K., \& Frahm, J. (1996). Somatotopic motor representation in the human anterior cerebellum. A high-resolution functional MRI study. Brain, 119, 1023-1029.

Pascual Leone, A., Dang, N., Cohen, L. G., Basil-Neto, J., Cammarota, A., \& Hallet, M. (1995). Modulation of motor responses evoked by transcranial magnetic stimulation during the acquisition of new fine motor skills. Journal of Neurophysiology, 74, 1034-1045.

Porro, C. A., Francescato, M. P., Cettolo, V., Diamond, M. E., Baraldi, P., Zuiani, Ch., Bazzocchi, M., \& di Prampero, P. E. (1996). Primary motor and sensory cortex activation during motor performance and motor imagery: A functional magnetic resonance imaging study. Journal of Neuroscience, 16, 7688-7698.

Pylyshyn, Z. W. (1981). The imagery debate: Analogue media versus tacit knowledge. Psychological Review, 88, 16-45.

Rao, S. M., Harrington, D. L., Haaland, K. Y., Bobholz, J. A., Coz, R. W., \& Binder, J. R. (1997). Distributed neural systems underlying the timing of movements. Journal of Neuroscience, $17,5528-5555$.

Roland, P. E., Larsen, B., Lassen, N. A., \& Skinhoj, E. (1980). Supplementary motor area and other cortical areas in organization of voluntary movements in man. Journal of Neurophysiology, 43, 118-136.

Roth, M., Decety, J., Raybaudi, M., Massarelli, R., Delon-Martin, Ch., Segebarth, Ch., Morand, S., Gemignani, A., Decorps, M., \& Jeannerod, M. (1996). Possible involvement of primary motor cortex in mentally simulated movement: A functional magnetic resonance imaging study. NeuroReport, 7 , 1280-1284.

Ryding, E., Decety, J., Sjöholm, H., Stenberg, G., \& Ingvar, D. H. (1993). Motor imagery activates the cerebellum re- 
gionally. A SPECT rCBF study with Tc-AHMPOA. Cognitive Brain Research, 1, 94-99.

Sabbah, P., Simond, G., Levrier, O., Habbib, M., Traboud, V., Murayama, N., Mazoyer, B. M., Briant, J. F., Rayboud, C., \& Salomon, G. (1995). Functional magnetic resonance imaging at $1.5 \mathrm{~T}$ during sensory motor and cognitive tasks. European Neurology, 35, 131-136.

Sakai, K., Takino, R., Hikosaka, O., Miyauchi, S., Sasaki, Y., Pütz, B., \& Fujimaki, N. (1997). Dissociation of neural correlates for motor execution and sensori-motor readiness within the cerebellum. Neuroimage, 5, 260.

Schlaug, G., Sanes, J. N., Thangaraj, V., Darby, D. G., Jäncke, L., Edelmann, R. R., \& Warach, S. (1996). Cerebral activation covaries with movement rate. NeuroReport, 7, 879-883.

Schnitzler, A., Salenius, S., Salmelin, R., Jousmaki, V., \& Hari, R. (1997). Involvement of primary motor cortex in motor imagery: A neuromagnetic study. Neuroimage, 6, 201-208.

Schupp, H. T., Lutzenberger, W., Birbaumer, N., Miltner, W., \&
Braun, Ch. (1994). Neurophysiological differences between perception and imagery. Cognitive Brain Research, 2, 7786.

Seitz, R., Canavan, A. G., Ya'güez, L., Herzog, H., Tellmann, L., Knorr, U., Huang, Y., \& Hömberg, V. (1997). Representations of graphomotor trajectories in the human parietal cortex: Evidence for controlled processing and automatic performance European Journal of Neuroscience, 9, 378-389.

Stephan, K. M., Fink, G. R., Passingham, R. E., Silbersweig, D., Ceballos-Baumann, O., Frith, C. D., \& Frackowiak, R. S. J. (1995). Functional anatomy of the mental representation of upper extremity movements in health subjects. Journal of Neurophysiology, 73, 373-386.

Strick, P. L., Hoover, J. E., \& Mushiake, H. (1993). Evidence for "output channels" in the basal ganglia and the cerebellum. In N. Mano, I. Hamada, \& M. R. DeLong (Eds.), Role of the cerebellum and the basal ganglia in voluntary movement (pp 171-180). New York: Elsevier. 